\title{
Mesh Deformations in X3D via CUDA with Freeform Deformation Lattices
}

\author{
Yvonne Jung, Holger Graf, Johannes Behr, and Arjan Kuijper \\ Fraunhofer IGD, Fraunhoferstraße 5, 64283 Darmstadt, Germany \\ \{yvonne.jung, holger.graf, johannes.behr, \\ arjan.kuijper\} aigd.fraunhofer.de
}

\begin{abstract}
In this paper we present a GPU-accelerated implementation of the well-known freeform deformation algorithm to allow for deformable objects within fully interactive virtual environments. We furthermore outline how our real-time deformation approach can be integrated into the X3D standard for more accessibility of the proposed methods. The presented technique can be used to deform complex detailed geometries without pre-processing the mesh by simply generating a lattice around the model. The local deformation is then computed for this lattice instead of the complex geometry, which efficiently can be carried out on the GPU using CUDA.
\end{abstract}

Keywords: Deformable objects, real-time simulation, FFD, CUDA, X3D.

\section{Introduction}

In this paper we present a hardware accelerated implementation of the freeform deformation (FFD) algorithm to allow for fully deformable objects in interactive virtual environments. The presented method can be used to deform complex detailed geometries without pre-processing the mesh by simply generating a lattice around the model. The local deformation is then computed for this lattice instead of the complex geometry, which efficiently is carried out on the GPU using CUDA. Freeform deformation is a common technique in computer graphics and image processing. In the field of medical imaging for instance, the grid-based FFD is used for registration approaches [2, 3], today's DCC tools (like e.g. 3ds Max [14]) utilize it as another modeling method, whereas we discuss the FFD in the field of object animation [4].

Despite the advancements in simulating deformable objects such as cloth $[12,15]$, hair [8, 17], or biological tissues [10, 11, 19] - not least because of the proliferation of multi-core CPUs and the rapid GPU development cycles [16] - it is still rather difficult, if possible at all, to integrate such techniques into interactive 3D applications like in Virtual or Mixed Reality environments [17, 20]. Hence, for ease of access, in this paper we will first discuss, how we have integrated this deformation method into X3D [9], an open ISO standard that not only defines a 3D interchange format, but which can also be used as a declarative application description language. The integration is achieved by means of a new physics material node type that is designed as a part of an object's general appearance. This idea is similar to the approach recently presented in [7], where the authors proposed an additional X3D node set that 
e.g. contains a special physics node for defining the physical properties associated with a shape node's geometry. Although these extensions originally were only intended for haptic rendering, they have the potential to support more dynamic and realistic object behaviors.

Next, we present and compare our implementation with focus on the various CUDA kernels used, while utilizing the OpenGL - CUDA interoperability as described in [5] for maximum performance. Here, we have basically implemented two different methods: the first one is based on the Bernstein polynomials as proposed in the original algorithm [1], while the other one utilizes a mass-spring-system that acts on the control points as described in [8]. Although the first method is not physicallybased, it is very fast, easy to implement even with CUDA, and allows achieving useful and interesting deformation effects during runtime, like elastic objects of arbitrary shapes that bounce in a plausible manner. Due to the inherent connectivity of the mass (or control) points in a mass-spring-system, the latter method is more intricate to implement on the GPU due to its SIMD architecture, but allows the application developer to design highly dynamic scenes.

To further optimize performance, we propose to use two different CUDA kernels. For objects with many vertices we can reach a full occupancy of all multiprocessors on the GPU. Therefore, vertex deformations are computed per thread: each thread needs all control points and the corresponding vertex. However, for low-poly objects only a few multiprocessors are used. Therefore, we will present our second approach based on parallel reduction. After that, we will present our runtime results and discuss some application examples, before we finally conclude the paper.

\section{Related Work}

In computer science there exist different methods to simulate deformable objects. Usually one distinguishes between physically-based deformation methods, like the finite element method (FEM), and geometrical ones. The latter only change the shape of polygonal models and are suitable as a tool for intuitive deformation tasks such as in CAD- or modeling-packages [14]. A well-known geometrical method is the gridbased free-form deformation. The original FFD method already was published by Sederberg and Perry 25 years ago [1], and is basically a geometric spline deformation based on the trivariate Bernstein polynomials [1, 18], which can be manipulated through a 3D lattice of control points, in which the geometries to be deformed are embedded. The idea is exemplarily visualized in Fig. 1 with a linear deformation.

However, other deformation schemes are possible, too, such as the mass-springbased simulation presented in [8], which combines this indirect deformation method with a more physically-based type of mesh deformation to animate complex hairstyles in real-time. Similarly, the SOFA framework for medical simulations is presented in [10]. Along with other grid-based deformation schemes a mass-spring-system is utilized for object deformations, which in a prototypical implementation is further accelerated with CUDA [6] by exploiting the highly parallel nature of modern graphics hardware. In [11], researchers from the same group lately also presented a method for simulating detailed geometric models using coarse grids by taking into 
account the topology and physical material of the embedded geometry to overcome problems like disconnected parts (with possibly different material properties) that are located within the same grid cell.

Generally, to achieve an animated deformation using physically-based methods, a simulation system needs to be advanced in time. Therefore, in [11] the implicit Euler integration scheme [12] is used to solve the motion equation for the given time step. The type of differential equation to be solved depends on the type of motion equation. Newtonian motion is based on Newton's second law of motion (i.e., $F=m \cdot a$ ) and can be solved by integrating an ordinary differential equation. The implementation is relatively easy because of the discretization to a finite number of mass points, and the integration is independent for each such particle.
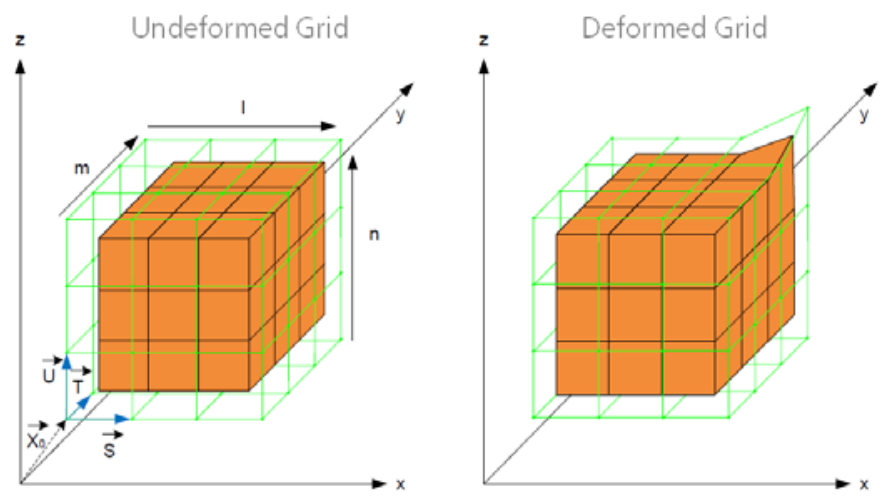

Fig. 1. Basic principle of grid deformation: on the left, exemplarily a cube embedded in a surrounding 3D lattice is shown, and on the right, the deformed grid/ cube is visualized

As already mentioned in the introduction, it is still rather difficult to integrate more advanced deformation techniques into interactive 3D applications like in VR or MR environments. Therefore, in [7] the authors proposed an additional X3D node set that, amongst others, contains a special physics node type for defining the tangible physical properties that are associated with a shape node's geometry (analogously to the X3D appearance node that is responsible for the optical properties of a certain geometry). Likewise, this physics node can be parameterized with various child nodes for specifying friction and so on. However, these extensions were only intended for haptic rendering, though they have the potential to support more realistic and physically-based object behaviors, e.g. in case of collisions with other scene objects. Similar in spirit, the VR/AR framework Instant Reality [13], which utilizes X3D as application description language, provides a "simulator" component that allows modeling simulation systems (e.g. for flow fields, particle dynamics or hair).

In this regard, X3D already provides a "rigid body physics" component, which describes how to model rigid bodies and their interactions, and a "particle systems" component, which specifies how to model particles and their interactions through the application of basic physics principles to affect the resulting motion [9]. However, simulated deformable objects are still not part of the current X3D standard. 


\section{Concept and Implementation}

In this section, we first describe the high-level concept, i.e. how we have integrated dynamic mesh deformations into X3D [9] with a new set of nodes for interactively controlling and parameterizing the desired deformations. After that, we explain our CUDA-based implementation to achieve better performance.

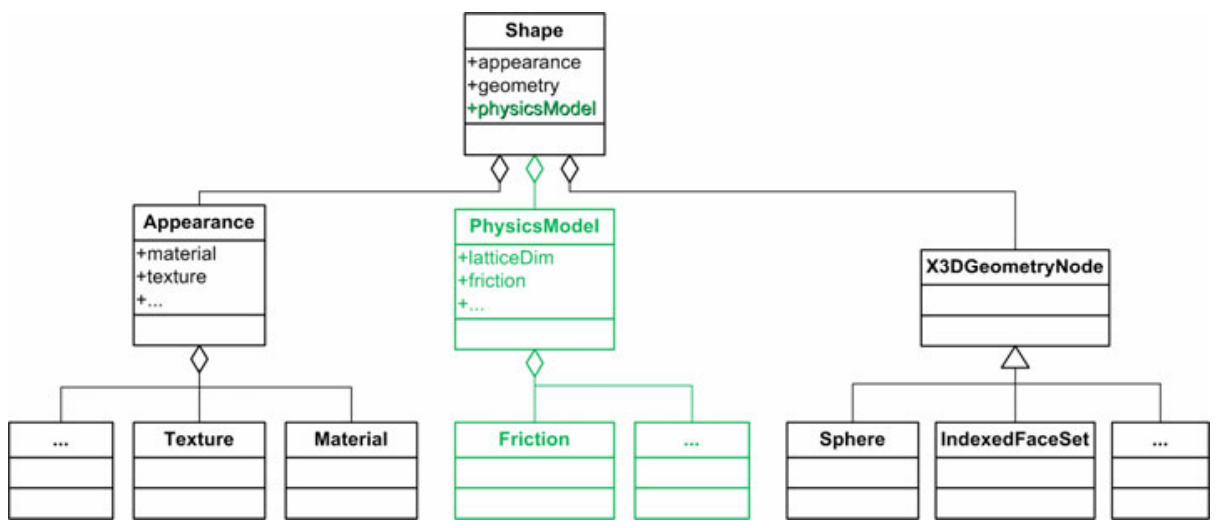

Fig. 2. Class diagram of the X3D Shape node: child nodes that are part of the X3D standard are shown in black, whereas the proposed new PhysicsModel node set is depicted in green

\subsection{Proposed X3D Node Extensions}

As already mentioned, our extension proposal basically follows the concepts as described by Wei et al. [7], though their focus was on enriching the standard X3D Shape node (which already aggregates the mesh geometry and appearance or optical properties - see Fig. 2) by function-defined geometry and tangible physical properties to allow for haptic exploration. Similar in spirit, with the ParticleSystem the X3D "particle systems" component defines a special type of shape node that specifies a complete particle system, since appearance and geometry both need to be controlled by the simulation to create plausible particle effects.

In contrast to the special case of particle physics [9], and following [7], we extend the standard shape node with a field 'physicsModel' for defining the object's physical properties in a composable way (as shown in the middle of Fig. 2), in order to support effects such as friction, restitution, or gravity and other external forces etc., which are not only useful for specifying deformation parameters but also for rigid body physics. If none of the PhysicsModel's child nodes are specified but a lattice dimension is set and some force is applied, the system defaults to geometric deformation.

\subsection{GPU-Based Implementation}

We use OpenGL and the interoperability with CUDA to implement the FFD on the GPU. The first step is to initialize OpenGL and load the mesh to a so-called VBO (Vertex Buffer Object), which conceptually is an array of bytes that can handle the 
upload of vertex attributes such as vertex positions, normals, colors and texture coordinates to the device memory of the graphics card. The deformation is then done within the rendering loop. In each iteration, the deformation is computed from the original object and the result is stored in a separate VBO, which we call Deformed Mesh VBO. Afterwards, the deformed mesh is used for display.

The lattice dimension is determined by the user manually via the SFVec $3 f$ field 'latticeDim' of the proposed PhysicsModel node (see greenish middle part of Fig. 2). All relevant lattice data is stored in a separate VBO. Based on the number of control points in each direction, the resulting object lattice is positioned automatically. The control points are arranged in equidistant steps (compare Fig. 1).

Moving a control point leads in turn to a deformation of the attached object (note that the mesh indices do not change during the deformation calculation). Therefore, the deformation function inside the rendering loop is called and the new positions of the mesh vertices are calculated, whereas this calculation depends on the chosen type of deformation. Currently, two deformation methods are provided: a geometrical one based on Bernstein polynomials [1] (outlined in sections 3.3 and 3.4) and a more physically-based that follows a mass-spring-system approach (see section 3.5).

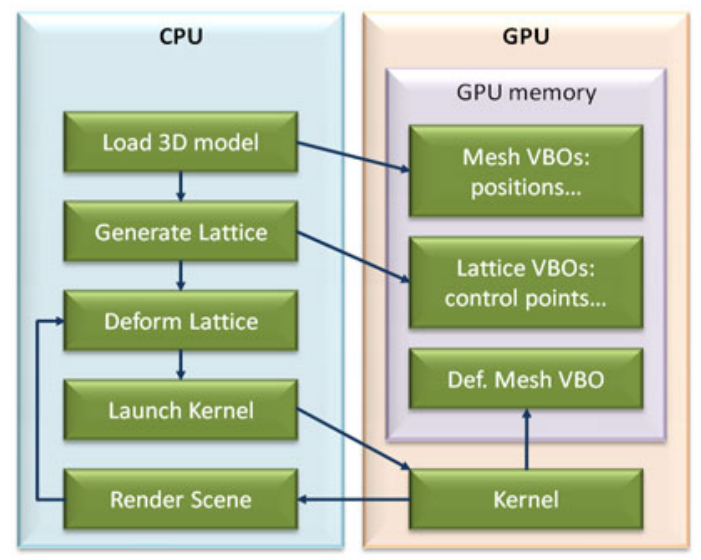

Fig. 3. Illustration of implementation concept (with CPU and GPU part)

In Fig. 3, the overall concept of the FFD implementation is coarsely sketched. Here, deforming the lattice means, that e.g. the user interactively changes the position of the control points (or respectively deforms the grid with a force $F$ ). Afterwards the CUDA kernel computes the FFD including the distance $d$ from each point $p$ to the surrounding control points. The weighted translation vector $f$ is added to $p$ and the result is then stored in the Deformed Mesh VBO.

The FFD itself is realized using CUDA. Before the data in the VBOs is usable, it has to be registered once as a CUDA resource. To gain access to the VBO data, a pointer to the memory location is needed. This is done by mapping the VBO resource to the CUDA address space. The result of the mapping is a pointer to the relevant data 
which can now be used in CUDA kernels. A more detailed description of the OpenGL - CUDA interoperability can be found in [5] on page $38-40$.

To further optimize performance we have implemented two different CUDA kernel approaches. One kernel is optimized for a low amount of vertices per object (about 500) and the other kernel is adjusted for objects with thousands of vertices.

\subsection{CUDA Kernel for Complex Objects}

For more complex high-resolution models we can easily reach a full occupancy of all multiprocessors (MP). Therefore, we compute the deformation of one vertex in one thread. Each thread needs all control points and the corresponding vertices. As mentioned in the previous section, we first focus on geometric mesh deformation methods, since these are much easier to parallelize. Hence, for each control point the Bernstein polynomial will be computed and therefore it is important, that the vertices as well as the control points are copied to shared memory. We choose a block size of $16 \times 16$. The first step is that each thread uploads one vertex to the shared memory. The vertices will be copied in a coalescing way, because 16 threads in a row copy vertices that are stored consecutively in the global memory.

In our approach the control points will not be copied to shared memory, because this will limit the lattice dimension and the occupancy of an MP can decrease. With a device of compute capability 1.3 (e.g. an NVidia GeForce GTX 295 graphics card) 1024 threads $\boldsymbol{t}$ can be hold by one multiprocessor. In our case four blocks will be assigned to one MP. The following equation shows, that $12 \mathrm{kB}$ of the shared memory $\boldsymbol{s}$ are used by the vertex data (where one vertex consists of three float values):

$$
\boldsymbol{s}=\boldsymbol{t} \cdot 3 \cdot \operatorname{sizeof}(\text { float })=1024 \cdot 3 \cdot 4 \text { Byte }=12 \mathrm{kB}
$$

The shared memory thus has a total amount of $16 \mathrm{kB}$. If a full occupancy of one multiprocessor is desired, then the maximum size of the lattice is 85 control points. To reduce the latency of the copy of the control points from the global memory, every thread loads identical control point data. This leads to broadcast memory accesses where the copy is fully parallelized. After copying the data, the FFD will be computed and the result is stored at the address of the Deformed Mesh VBO.

\subsection{CUDA Kernel for Simple Objects}

When an object with only a few vertices shall be deformed, it is not ideal to use the CUDA kernel for complex objects. Assume that we have an object with 512 vertices. Then there will be only two blocks with $16 \times 16$ threads and only two multiprocessors of the GPU will be used. To alleviate this issue we compute the deformation of one vertex in one block. Each thread in a block will compute one "sub" deformation. This means that a thread computes one Bernstein polynomial of one control point. Therefore, each thread uploads a control point to shared memory and computes a sub deformation. This sub deformation must then be summed up to get the complete deformation. This is done with the help of parallel reduction. To do the parallel reduction, similar to the FFT algorithm in signal processing, the thread count of one block must be the next power of two. A disadvantage of this technique is that the 
lattice can only have as much control points as the maximum count of threads per block, but in general the lattice for small objects has much less control points.

\subsection{Dynamics Simulation}

In order to simulate elastic objects, the other method is based on a mass-springsystem that acts on the lattice, as originally proposed in [8] for hair simulation. In our first prototypical implementation we simply identify Newton's second law of motion with Hooke's law of elasticity (i.e., $F=D \cdot \Delta l$ ). Then, the forces between all control points are updated in each frame and applied to the control points. To parallelize this approach all possible forces between the control points are independently computed by following the ping-pong "rendering" approach (via a helper buffer) as known from GPGPU programming. Afterwards, each control point is handled by a thread that computes the movement, which results in the final deformation.

\section{Discussion and Results}

We have implemented our FFD approach using CUDA and C++ and prototypically integrated the proposed node set into the MR framework Instant Reality [14]. First, we compared the computation time on the CPU with both approaches for simple and complex objects with a different count of vertices for a wireframe goblet model. In Table 1 the results are shown - all measurements are done with a lattice of $3 \times 3 \times 3$. For a small number of vertices there is not much speed up. If the object has less than 5,000 vertices, a slight speed up can be achieved when using the CUDA kernel for simple objects. If the number of vertices reaches 10,000 vertices and more, the computation time of the CPU is very high. With the CUDA kernel for complex objects we can achieve a considerably smaller computation time of the FFD.

Table 1. Runtime comparison of both CUDA kernels vs. computation time on CPU

\begin{tabular}{rrrr}
\hline \#vertices & CPU time/ $\mathbf{m s}$ & $\begin{array}{l}\text { GPU time/ ms } \\
\text { (kernel f. simple obj.) }\end{array}$ & \multicolumn{2}{l}{$\begin{array}{l}\text { GPU time/ ms } \\
\text { (kernel f. complex obj.) }\end{array}$} \\
\hline 502 & 7.5 & 4.0 & 15.1 \\
\hline 3099 & 47.6 & 5.2 & 15.4 \\
\hline 34,834 & 526.0 & 39.5 & 15.9 \\
\hline
\end{tabular}

Fig. 4 shows the deformation of two different 3D objects: a simple point cloud given as X3D PointSet node (left) and a dino model given as IndexedFaceSet nodes (right). The grid's control points are visualized as blue spheres, which for testing purposes can be moved to trigger the deformation process. Table 2 shows the runtime results obtained from both test sets shown in Fig. 4. For comparison, a different hardware setup was chosen here. Instead of the Core2 Quad CPU @ $2.66 \mathrm{GHz}$ (where obviously only one core was active) and a GPU with compute capability 1.3 - as used for the benchmarks in Table 1 - a single core CPU and a caps 1.0 GPU was used. 
Table 2. Benchmark (Pentium IV @ 3.2 GHz CPU with GeForce 8800 GTX GPU)

\begin{tabular}{rrrrr}
\hline Model & \#vertices & latticeDim & CPU time/ ms & GPU time/ ms \\
\hline Point cloud & 5,000 & $3 \times 3 \times 3$ & 15 & 2.7 \\
\hline$"$ & 10,000 & $3 \times 3 \times 3$ & 47 & 5.4 \\
\hline$"$ & 10,000 & $4 \times 4 \times 4$ & 94 & 10.7 \\
\hline$"$ & 10,000 & $5 \times 5 \times 5$ & 203 & 34.5 \\
\hline$"$ & 10,000 & $6 \times 6 \times 6$ & 359 & 162.8 \\
\hline$"$ & 50,000 & $3 \times 3 \times 3$ & 219 & 26.9 \\
\hline Dino & 8,145 & $7 \times 7 \times 7$ & 484 & 207.0 \\
\hline
\end{tabular}
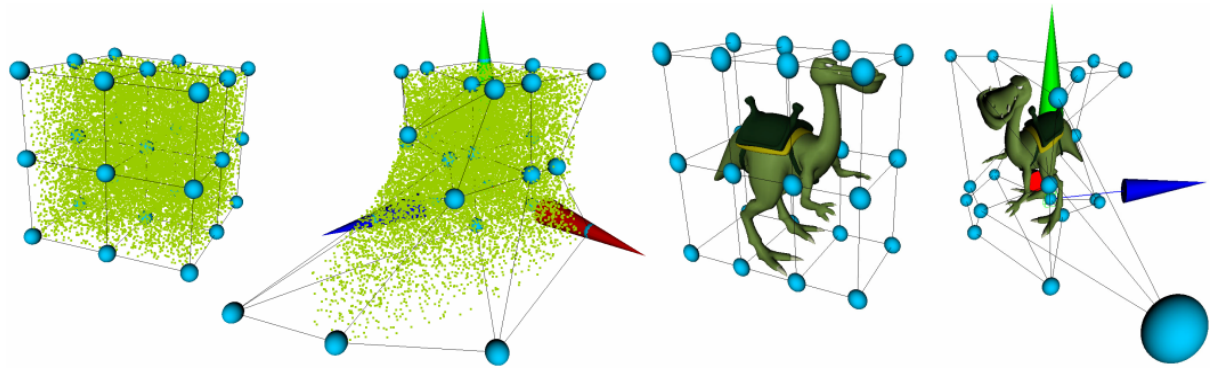

Fig. 4. Left: PointSet node with 15,000 test points and $3 \times 3 \times 3$ control points (visualized as blue spheres). Right: Dino model with $2 \times 3 \times 4$ control points (the three colored cones in the deformed image denote manipulation handles). Both are shown undeformed and deformed.

\section{Conclusions}

In this paper we have presented a hardware accelerated implementation of the freeform deformation algorithm to allow for fully deformable objects within interactive virtual environments. Moreover, we have described how an integration of the presented deformation approach into the current X3D standard can look like to improve the accessibility of the proposed methods also application developers that are no experts in this area. These techniques can be used to easily deform complex geometries without pre-processing the mesh by automatically generating a lattice around the model. The local deformation is then computed for this grid instead of the original geometry, which efficiently can be done on the GPU using CUDA.

Another benefit is the generalization of physical properties that are inherently associated with a certain 3D object, which can also be used to define the respective parameters for both already existing X3D physics component. However, a drawback of our CUDA-based implementation is that high-end graphics hardware (i.e. a modern Shader Model 4.0/ 5.0 GPU) is required. 


\section{References}

1. Sederberg, T.W., Parry, S.R.: Free-Form Deformation of Solid Geometric Models. In: ACM SIGGRAPH 1986, vol. 20, pp. 151-160 (1986)

2. Rueckert, D., Sonoda, L.I., Hayes, C., Hill, D.L.G., Leach, M.O., Hawkes, D.J.: Nonrigid Registration Using Free-Form Deformations: Application to Breast MR Images. IEEE Transactions on Medical Imaging 18, 712-721 (1999)

3. Modat, M., Ridgway, G.R., Taylor, Z.A., Lehmann, M., Barnes, J., Hawkes, D.J., Fox, N.C., Ourselin, S.: Fast Free-Form Deformation using Graphics Processing Units. Computer Methods and Programs in Biomedicine (2009)

4. Faloutsos, P., van de Panne, M., Terzopoulos, D.: Dynamic Free-Form Deformations for Animation Synthesis. IEEE Transactions on Vis. and Comp. Graphics 3 (1997)

5. NVIDIA, CUDA Programming Guide, Edition 3.0 (2010)

6. Sanders, J., Kandrot, E.: CUDA by example: an introduction to general-purpose GPU programming. Addison Wesley, Reading (2010)

7. Wei, L., Sourin, A., Stocker, H.: Function-based haptic collaboration in X3D. In: Proc. of Web3D 2009, pp. 15-23. ACM, New York (2009)

8. Volino, P., Magnenat-Thalmann, N.: Animating complex hairstyles in real-time. In: VRST 2004: ACM Symposium on VR Software \& Technology, pp. 41-48. ACM, NY (2004)

9. Web3D Consortium. X3D (2008), http: / / www . web3d.org/x3d/specifications /

10. Allard, J., Cotin, S., Faure, F., Bensoussan, P.-J., Poyer, F., Duriez, C., Delingette, H., Grisoni, L.: SOFA - an Open Source Framework for Medical Simulation. In: Medicine Meets Virtual Reality (MMVR 15), Long Beach, USA, pp. 13-18 (2007)

11. Nesme, M., Kry, P.G., Jerábková, L., Faure, F.: Preserving topology and elasticity for embedded deformable models. In: SIGGRAPH 2009 Papers, pp. 1-9. ACM, New York(2009)

12. Witkin, A., Baraff, D.: Large steps in cloth simulation. Computer Graphics 32, 43-54 (1998)

13. Fraunhofer IGD, Instant Reality framework (2011), http: / / www. instantreality.org/

14. Autodesk, 3ds Max (2011), http: / / area.autodesk.com/3dsmax2011/features

15. Knuth, M., Kohlhammer, J., Kuijper, A.: Embedding Hierachical Deformation within a Realtime Scene Graph - A Simple Approach for Embedding GPU-based Realtime Deformations using Trilinear Transformations Embedded in a Scene Graph. In: GRAPP 2010, pp. 246-253 (2010)

16. Binotto, A.P.D., Daniel, C., Weber, D., Kuijper, A., Stork, A., Pereira, C., Fellner, D.W.: Iterative SLE Solvers over a CPU-GPU Platform. In: HPCC 2010, pp. 305-313 (2010)

17. Jung, Y., Knöpfle, C.: Real Time Rendering and Animation of Virtual Characters. IJVR 6(4), 55-66 (2007)

18. Kalbe, T., Koch, T., Goesele, M.: High-Quality Rendering of Varying Isosurfaces with Cubic Trivariate-Continuous Splines. In: ISVC 2009, pp. 596-607 (2009)

19. Kuijper, A., Heise, B.: An automatic cell segmentation method for differential interference contrast microscopy. In: ICPR 2008, pp. 1-4 (2008) 